\title{
Clinical Impact of Carotid Plaque Score rather than Carotid Intima- Media Thickness on Recurrence of Atherosclerotic Cardiovascular Disease Events
}

\author{
Hayato Tada, Tamami Nakagawa, Hirofumi Okada, Takuya Nakahashi, Mika Mori, Kenji Sakata, Atsushi \\ Nohara, Masayuki Takamura and Masa-aki Kawashiri
}

Department of Cardiology, Kanazawa University Graduate School of Medicine, Kanazawa, Japan

Aim: Carotid plaque score (cPS) reflecting throughout the carotid artery plaque burden may be a better marker than carotid intima-media thickness (cIMT) is. We aimed to compare the prognostic utility of these measurements in patients with atherosclerotic cardiovascular disease (ASCVD).

Methods: We retrospectively examined 2,035 Japanese patients with ASCVD who underwent carotid ultrasonography between January 2008 and December 2015 at Kanazawa University Hospital. Median follow-up period was 4 years. We used Cox models that adjusted for established risk factors of ASCVD, including age, gender, hypertension, diabetes, smoking, and serum lipids to assess the association of cIMT as well as cPS with major adverse cardiac events (MACE). MACE was defined as all-cause mortality or rehospitalization for a cardiovascular-related illness.

Results: During follow-up, 243 participants experienced MACE. After adjustment for established risk factors, cPS was associated with MACE (hazard ratio $[\mathrm{HR}]=3.38$ for top quintile vs. bottom quintile of cPS; $95 \%$ confidence interval $[\mathrm{CI}] 1.82-6.27 ; P$ trend $<0.001)$, while cIMT was not $(\mathrm{HR}=0.88, P=0.57)$. Addition of the cPS to established risk factors significantly improved risk discrimination $(\mathrm{C}$-index 0.726 vs. $0.746 ; P=0.017)$.

Conclusion: These results suggest that cPS, rather than cIMT may be a better marker to identify increased risk for recurrence of MACE among patients with secondary prevention setting.

See editorial vol. 27: 6-7

Key words: Atherosclerotic cardiovascular disease, Carotid ultrasonography, Risk stratification, Secondary prevention

\section{Introduction}

Although the prognosis after suffering from atherosclerotic cardiovascular disease (ASCVD) has been improved with the introduction of primary coronary angioplasty as well as medical treatment such as LDLlowering therapies and anti-platelet therapies ${ }^{1-3)}$, secondary prevention of these patients is still one of the major challenges for cardiologists. Identifying individuals at the higher risk for recurrent cardiovascular events and, then, aggressively addressing modifiable risk factors in these individuals may be beneficial regarding health outcomes and cost.
Carotid intima-media thickness (cIMT) determined by less-invasive ultrasonography has been shown to be a surrogate marker for coronary atherosclerosis ${ }^{4)}$, and increased cIMT has been shown to be associated with future cardiovascular events ${ }^{5-7)}$. On the other hand, carotid plaque score (cPS) reflecting throughout the carotid artery plaque burden may be better marker. Our previous study has shown that the carotid plaque score (cPS), reflecting the cumulative atherosclerotic burden of the carotid artery, is a more useful parameter to predict the atherosclerotic severity of the coronary artery than cIMT in patients with familial hypercholesterolemia $(\mathrm{FH})^{8)}$. Some previous

Address for correspondence: Hayato Tada, Department of Cardiology, Kanazawa University Graduate School of Medicine 13-1 Takara-machi, Kanazawa, 9208640, Japan E-mail: ht240z@sa3.so-net.ne.jp

Received: February 28, 2019 Accepted for publication: March 25, 2019

Copyright@2020 Japan Atherosclerosis Society

This article is distributed under the terms of the latest version of CC BY-NC-SA defined by the Creative Commons Attribution License. 
studies have shown that cPS or carotid plaque burden was better marker for severity of coronary artery disease or the first cardiovascular events among primary prevention group ${ }^{9,10)}$. However, few data exist regarding the clinical impact of cPS on the recurrence of ASCVD, comparing that of cIMT in a setting of secondary prevention. Accordingly, we aimed to compare the prognostic utility of these measurements among patients with ASCVD. Moreover, such modality could help us to estimate when and how rapidly coronary atherosclerosis in patients with ASCVD develop ${ }^{8,11)}$. Accordingly, we aimed (1) to compare the prognostic utility of these measurements and (2) to estimate the onset and progression of carotid atherosclerosis in patients of a secondary prevention setting.

\section{Materials and Methods}

\section{Study Subjects}

In total, 2,132 consecutive patients who had any histories of ASCVD events and underwent carotid ultrasonography between January 2006 and December 2015 were retrospectively analyzed. Among the 2,132 patients, 97 patients lacking data $(5 \%)$ were excluded (Supplemental Fig. 1). Thus, 2,035 patients with ASCVD whose ages ranged from 14 to 95 years were included in the analysis (male $=1,253$, mean age $=65$ \pm 12 years, mean LDL cholesterol $=115 \pm 36 \mathrm{mg} / \mathrm{dL}$ ).

\section{Clinical Evaluations}

Blood samples were drawn for assays after overnight fasting. Serum levels of total and HDL cholesterol and triglycerides were enzymatically determined (Qualigent, Sekisui Medical, Tokyo, Japan) using automated instrumentation based on previously described assays ${ }^{12)}$. Hypertension was defined as systolic blood pressure $\geq 140 \mathrm{mmHg}$ and/or diastolic blood pressure $\geq 90 \mathrm{mmHg}$ or treatment with antihypertensive medications. The presence of diabetes was defined as described previously by the Japan Diabetes Society ${ }^{13)}$. Smoking status was defined as current smoking habits. Left ventricular ejection fraction (LVEF) was calculated either by modified Simpson's method or by Teichholz method. We defined major adverse cardiac events (MACE) as either all-cause death, including cardiac death, ST elevated myocardial infarction (STEMI), non-ST elevated myocardial infarction (NSTEMI), unstable angina pectoris (UAP), staged percutaneous coronary intervention (PCI) or coronary artery bypass grafting (CABG), ischemic as well as hemorrhagic stroke, heart failure requiring hospital admission.

\section{Carotid Ultrasonography}

The parameters for carotid ultrasonography were measured using the Aplio carotid ultrasonography machine (Toshiba Medical Systems, Tokyo, Japan) with a $7.5-\mathrm{MHz}$ transducer by trained sonographers who were blinded to the clinical data. cIMT was recorded during ultrasonography as described previously ${ }^{14)}$. In brief, cIMT from the right and left sides was measured from the far wall, the location of which was identified as the vertical distance from the leading edge of the first to the second echogenic line. Three cIMT determinations were measured in the walls at the site of the greatest thickness for each common carotid artery, and these measurements of both the arteries were averaged and expressed as the mean cIMT. In addition, cPS was computed by summing the maximal thickness of plaques, that were defined as focal intima-media thickening $\geq 1.1 \mathrm{~mm}$, in each segment on both sides $(a+b+c+$ thickness of the contralateral plaques in each segment on both sides) as described previously ${ }^{8,15)}$.

\section{Ethical Consideration}

The Ethics Committee of Kanazawa University approved this study. All procedures followed were in accordance with the ethical standards of the responsible committee on human experimentation (institutional and national) and with the Helsinki Declaration of 1975 , revised in 2008. Informed consent was obtained from all the subjects for inclusion in the study.

\section{Statistical Analysis}

Continuous variables are presented as mean \pm standard deviation, and categorical variables are expressed as counts and percentages. For values lacking a normal distribution, the median and interquartile range are reported. Mean values of continuous variables were compared using Student's $t$-test for independent data, and median values were compared using the nonparametric Mann Whitney $U$-test/Wilcoxon rank-sum test. Categorical variables were compared using the chi-square test. Hazard ratios were determined adjusted for age, sex, hypertension, current smoking, triglyceride, LDL cholesterol, HDL cholesterol, anti-platelet therapy, and lipid-lowering therapy. Hazard ratios for occurrence of endpoint events were determined for each quintile of cPS, with quintile 1 serving as the referent group. The cumulative fraction of events was estimated as 1 minus the Kaplan-Meier estimate of survival free of event. The differences of the cumulative fraction of events between subgroups were assessed by the log-rank test. Receiver-operating characteristic analysis was per- 
Table 1. Baseline characteristics

\begin{tabular}{|c|c|c|c|c|}
\hline \multirow[t]{2}{*}{ Variable } & \multirow{2}{*}{$\begin{array}{c}\text { All } \\
(n=2,035)\end{array}$} & \multicolumn{2}{|c|}{ MACE } & \multirow[t]{2}{*}{$p$ value } \\
\hline & & YES $(n=243)$ & $\mathrm{NO}(n=1,792)$ & \\
\hline Age (years) & $65 \pm 12$ & $69 \pm 9$ & $65 \pm 13$ & $<0.001$ \\
\hline Men & $1,253(62 \%)$ & $187(77 \%)$ & $1,066(59 \%)$ & $<0.001$ \\
\hline Hypertension & $1,459(72 \%)$ & $220(91 \%)$ & $1,239(69 \%)$ & $<0.001$ \\
\hline Diabetes & $841(41 \%)$ & $138(57 \%)$ & $703(39 \%)$ & $<0.001$ \\
\hline Smoking & $1,122(55 \%)$ & $196(81 \%)$ & $926(52 \%)$ & $<0.001$ \\
\hline Total cholesterol (mg/dL) & $192 \pm 42$ & $180 \pm 40$ & $194 \pm 42$ & $<0.001$ \\
\hline Low-density lipoprotein cholesterol (mg/dL) & $115 \pm 36$ & $108 \pm 34$ & $116 \pm 37$ & 0.004 \\
\hline High-density lipoprotein cholesterol (mg/dL) & $52 \pm 16$ & $46 \pm 13$ & $53 \pm 16$ & $<0.001$ \\
\hline Triglyceride (mg/dL) & $106[75-154]$ & $108[75-156]$ & $106[73-154]$ & 0.79 \\
\hline Anti-platelet therapy & $1,983(97 \%)$ & $241(99 \%)$ & $1,742(97 \%)$ & 0.142 \\
\hline Lipid-lowering therapy & $1,846(91 \%)$ & $236(97 \%)$ & $1,610(90 \%)$ & $<0.001$ \\
\hline Carotid IMT (mm) & $0.8[0.7-1.0]$ & $1.0[0.8-1.1]$ & $0.8[0.7-1.0]$ & $<0.001$ \\
\hline Carotid plaque score & $7.4[3.4-12.3]$ & $12.2[8.0-16.5]$ & $6.8[3.1-11.4]$ & $<0.001$ \\
\hline
\end{tabular}

MACE: major adverse cardiac events, IMT: intima-media thickness

formed and C-statistic was calculated to estimate the predictive performance of the evaluated parameters. C-statistic estimates were compared using the method of DeLong et al. Intraobserver/interobserver variability between sonographers was assessed using the BlandAltmann method, and coefficient of variation $(\mathrm{CV})$ with 40 randomly selected subjects. A $p$ value of $<$ 0.05 was considered statistically significant. Statistical analysis was performed using $\mathrm{R}$ version 3.3.2.

\section{Results}

\section{Reproducibility of Measurements}

Supplemental Fig. 2 and Supplemental Fig. 3 show intra- and interobserver reproducibility for measurements of cIMT and cPS. Bland-Altman analysis demonstrated good agreements between both within intraobserver with a CV of $9.1 \%, 10,4 \%$ and within interobserver with a CV of $9.9 \%, 11.1 \%$ for measurements cIMT, and cPS, respectively.

\section{Baseline Characteristics}

Table 1 illustrates the baseline characteristics of the study population. We observed 243 MACE events during the median 4 years of follow-up period. Table 2 illustrates details of MACE. As expected, the patients with MACE were significantly older than those without ASCVD. Frequencies of other traditional risk factors such as male gender, hypertension, diabetes, and smoking habits were significantly higher and that of HDL cholesterol was significantly lower in patients with MACE than in those without. Under these conditions, cIMT and cPS were significantly higher in patients with MACE than in those without.
Table 2. Type of MACE

\begin{tabular}{lc}
\hline Type of MACE & Number (\%) \\
\hline All-cause death & $89(36 \%)$ \\
death associated with ASCVD & $53(22 \%)$ \\
death not-associated with ASCVD & $36(15 \%)$ \\
Cardiovascular disease & $89(37 \%)$ \\
STEMI & $7(3 \%)$ \\
NSTEMI & $17(7 \%)$ \\
UAP & $18(7 \%)$ \\
staged PCI/CABG & $47(19 \%)$ \\
Stroke & $19(8 \%)$ \\
ischemic stroke & $14(6 \%)$ \\
hemorrhagic stroke & $5(2 \%)$ \\
Heart failure & $46(19 \%)$ \\
\hline
\end{tabular}

MACE: major adverse cardiac events, ASCVD: atherosclerotic cardiovascular disease, STEMI: ST-elevated myocardial infarction, NSTEMI: non ST-elevated myocardial infarction, UAP: unstable angina pectoris, PCI: percutaneous coronary intervention, CABG: coronary artery bypass graft

Fig. 1 illustrates distribution of cIMT as well as cPS.

\section{Factors Associated with MACE}

Next, we investigated the factors associated with MACE, including carotid ultrasonographic parameters (Table 3). cPS was significantly associated with MACE $(\mathrm{HR}=1.08,95 \% \mathrm{CI}, 1.04-1.12, p<0.001)$ after adjustment for established risk factors including age, gender, hypertension, diabetes, smoking, triglyceride, LDL cholesterol, HDL cholesterol, and LVEF, while cIMT was not associated with MACE $(\mathrm{HR}=$ $0.89,95 \%$ CI, $0.52-1.49, p=0.61$ ). 

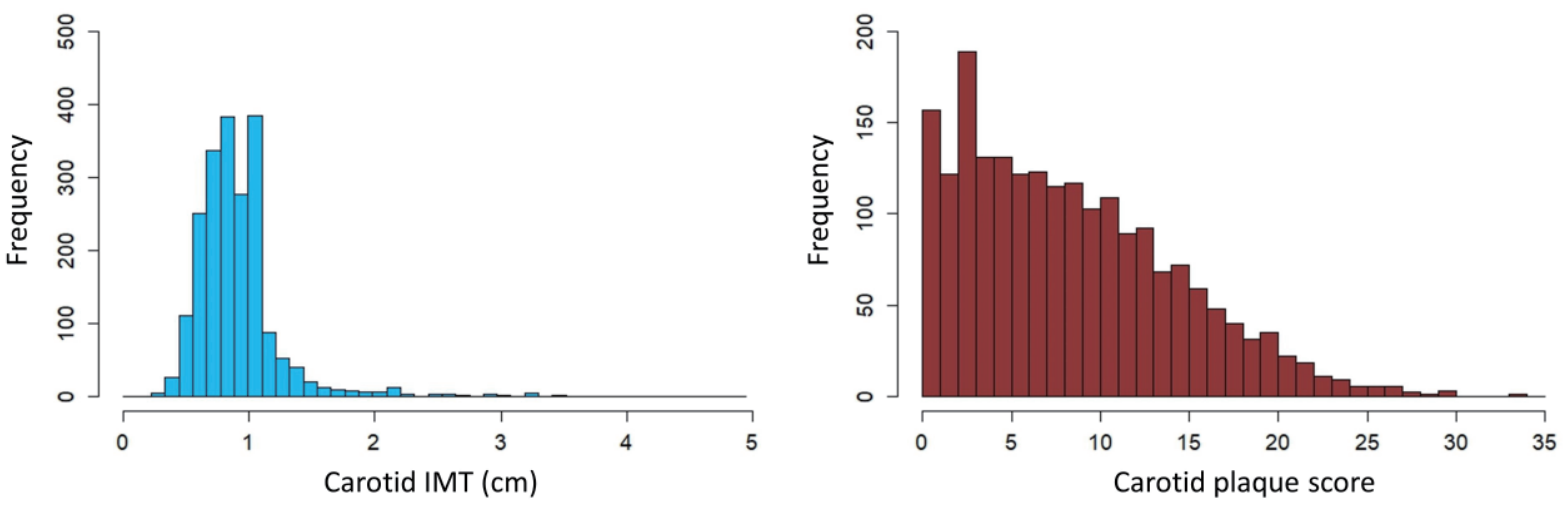

Fig. 1. Distribution of carotid IMT and carotid plaque score

(A) X-axis represents carotid IMT (cm). Y-axis represents frequency.

(B) X-axis represents carotid plaque score $(\mathrm{cm})$. Y-axis represents frequency.

Table 3. Factors associated with MACE

\begin{tabular}{lcc}
\hline Variables & HR $(95 \% \mathrm{CI})$ & $p$-value \\
\hline Age & $1.017(1.00-1.03)$ & 0.02 \\
Gender & $1.41(1.04-1.85)$ & 0.007 \\
Hypertension & $1.49(1.09-2.16)$ & 0.004 \\
Diabetes & $1.20(0.90-1.74)$ & 0.18 \\
Smoking & $1.85(1.25-2.70)$ & 0.002 \\
Triglyceride & $0.99(0.99-1.00)$ & 0.027 \\
LDL cholesterol & $0.996(0.99-1.00)$ & 0.09 \\
HDL cholesterol & $0.95(0.90-1.00)$ & 0.002 \\
Anti-platelet therapy & $1.28(0.91-1.80)$ & 0.21 \\
Lipid-lowering therapy & $0.91(0.63-1.28)$ & 0.55 \\
LVEF & $1.03(1.01-1.05)$ & 0.021 \\
Carotid IMT & $0.89(0.52-1.40)$ & 0.61 \\
Carotid plaque score & $1.08(1.04-1.12)$ & $<0.001$ \\
\hline
\end{tabular}

MACE: major adverse cardiac events, LVEF: left ventricular ejection fraction, IMT: intima-media thickness

Table 4. Impact of carotid plaque score on MACE

\begin{tabular}{|c|c|c|c|c|c|c|}
\hline & \multicolumn{5}{|c|}{ Carotid plaque score Quintile } & $P_{\text {trend }}$ \\
\hline \multicolumn{7}{|l|}{ MACE } \\
\hline Number (Events) & $421(20)$ & $408(21)$ & $402(38)$ & $407(57)$ & 407 (107) & \\
\hline HR (95\%CI) & Reference & $0.80(0.41-1.57)$ & $1.47(0.81-2.67)$ & $1.84(1.01-3.36)$ & $3.38(1.82-6.27)$ & $<0.001$ \\
\hline
\end{tabular}

cPS: carotid plaque score, MACE: major adverse cardiac events, HR: Hazard ratio, Q: quintile

The Power of cPS for Predicting MACE

When the patients were divided into 5 groups based on cPS quintiles, those with the highest cPS had 3.38 fold greater risk of MACE than those with lowest quintile (95\%CI $1.82-6.27, p<0.001$ vs. lowest cPS, Table 4). Moreover, cumulative MACE rate increased according to quintile of the cPS (Log-rank trend, $p<$ 0.001 , Fig. 2). 


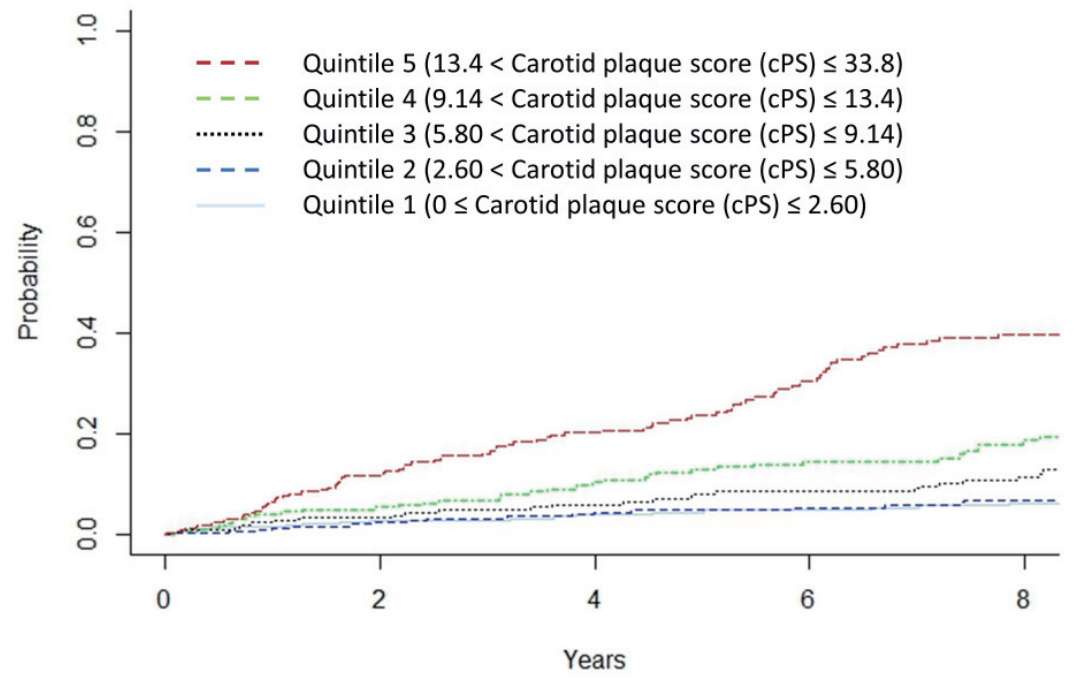

Fig. 2. Cumulative event rate divided by carotid plaque score quintile

$\mathrm{X}$-axis represents follow-up period (year). Y-axis represents event rate.

Red dotted line indicates quintile 5. Green dotted line indicates quintile 4. Black dotted line indicates quintile 3. Blue dotted line indicates quintile 2. Light blue solid line indicates quintile 1.

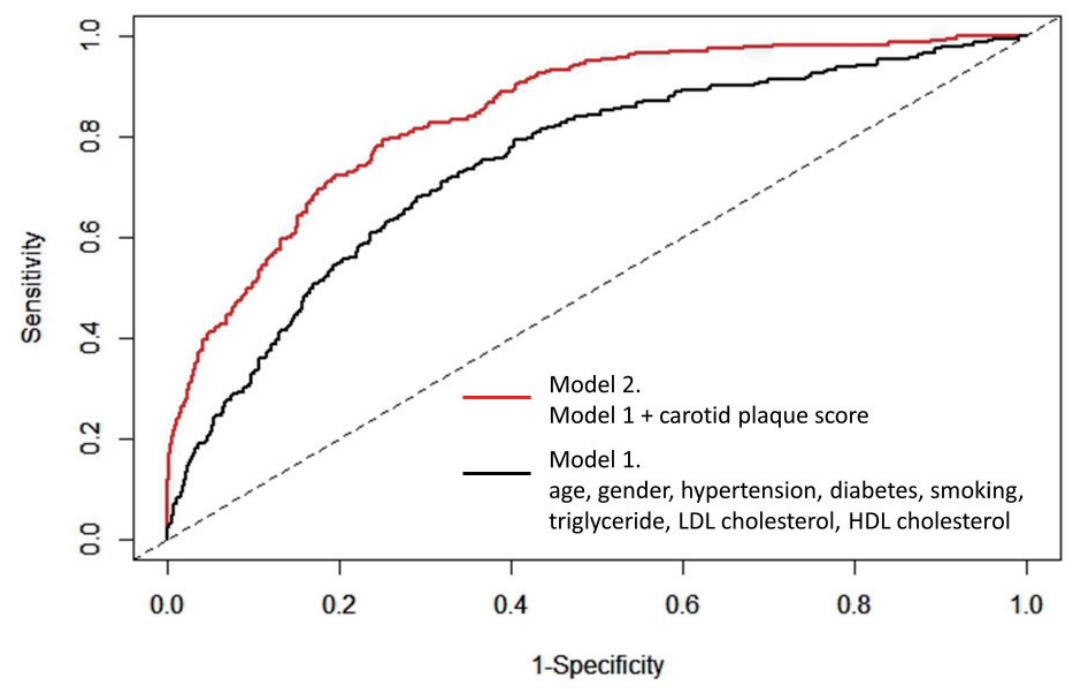

Fig. 3. Receiver-operating characteristic curve

The black line (model 1) indicates the receiver-operating characteristic curve using traditional risk factors, including age, gender, hypertension, diabetes, smoking, triglyceride, LDL cholesterol, HDL cholesterol. The red line (model 2) indicates the receiver-operating characteristic curve using traditional risk factors and carotid plaque score.

\section{Risk Discrimination by cPS}

We investigated whether the discrimination of a model based on the established traditional risk factors, including age, gender, hypertension, diabetes, smoking, triglyceride, LDL cholesterol, and HDL cholesterol differed from that of a model that also included cPS. The C-statistic for the traditional risk factors model was 0.726 , which increased to 0.746 (Fig. 3, $p$
$=0.01748)$ after the addition of cPS information to the model. To assess the discriminatory potential of these parameters, we also assessed the continuous net reclassification improvement (NRI) and integrated discrimination improvement (IDI). We found that the addition of cIMT or $\mathrm{CPS}$ to the traditional risk factor model improved reclassification (continuous NRI = $0.227,95 \% \mathrm{CI}=0.106-0.358, p<0.001 ; \mathrm{IDI}=$ 
Table 5. Risk discrimination by carotid intima-media thickness or carotid plaque score beyond traditional risk factors

\begin{tabular}{lcccc}
\hline & NRI $(95 \%$ CI $)$ & \multicolumn{1}{c}{$p$ value } & IDI $(95 \%$ CI $)$ & $p$ value \\
\hline TRF & Reference & Reference & \\
TRF + cIMT & $0.227(0.106-0.358)$ & $<0.001$ & $0.0103(0.0021-0.0365)$ & 0.048 \\
TRF + cPS & $0.481(0.234-0.733)$ & $<0.001$ & $0.021(0.0051-0.102)$ & $<0.001$ \\
\hline
\end{tabular}

CI, confidence interval; HR, hazard ratio; NRI, net reclassification improvement; IDI, integrated discrimination improvement; TRF, traditional risk factors (age, gender, hypertension, diabetes, smoking, triglyceride, LDL cholesterol, and HDL cholesterol)
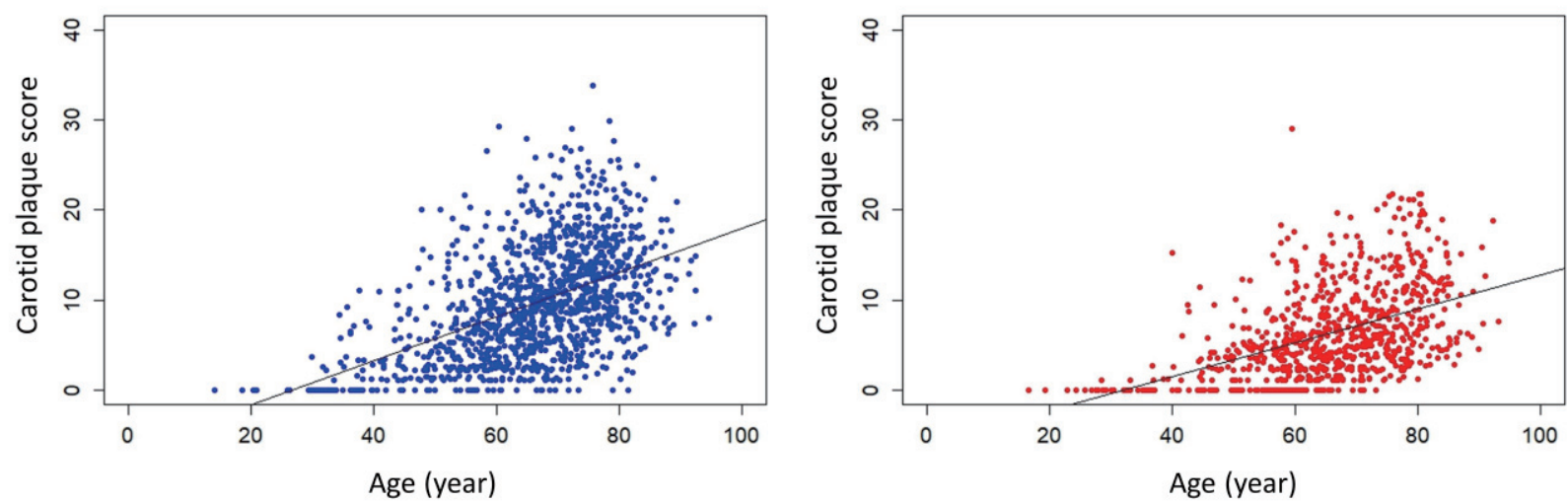

Fig. 4. Plots of correlation between age $(\mathrm{X})$ and carotid plaque score $(\mathrm{Y})$ in male $(\mathrm{A})$ and female (B) patients with ASCVD

The regression equations were $\mathrm{Y}=0.245 \mathrm{X}-6.63(r=0.50, p<0.001)$ in male and $\mathrm{Y}=0.189 \mathrm{X}-6.09(r=0.469, p<0.001)$ in female.

$0.0103,95 \% \mathrm{CI}=0.0021-0.0365, p=0.048$; continuous $\mathrm{NRI}=0.481,95 \% \mathrm{CI}=0.234-0.733, p<0.001$; IDI $=0.021,95 \% \mathrm{CI}=0.0051-0.102, p<0.001$, Table 5).

\section{Development of Carotid Plaque}

Finally, we evaluated the correlation coefficient between age and cPS in each gender (Fig.4). The regression equations were $\mathrm{Y}=0.245 \mathrm{X}-6.63(r=0.50, p$ $<0.001)$ in male and $\mathrm{Y}=0.189 \mathrm{X}-6.09(r=0.469, p<$ $0.001)$ in female. These results suggest that carotid atherosclerosis had already started to develop, on average, at 27 and 32 years of age in male and female patients with ASCVD, respectively.

\section{Discussion}

The present study compared the clinical utility of 2 different measurements obtained from carotid ultrasonography in patients with ASCVD. This study provides the following important evidence: (1) cPS, rather than cIMT is an independent predictor of MACE (2) cPS is an useful clinical tool for risk strati- fication (3) On average, carotid atherosclerosis may start to develop at 27 and 32 years of age in male and female patients in a secondary prevention setting, respectively.

Despite the significantly elevated cardiovascular disease risk in patients of a secondary prevention setting, their individual risk is heterogeneous ${ }^{16-19)}$. Further risk stratification strategies would be needed to pin-point extreme high-risk individuals. We have shown previously that plaque burden in coronary arteries assessed by coronary computed tomography angiography was associated with future ASCVD events in patients with $\mathrm{FH}^{11)}$. In this study, cPS which could be assessed non-invasively, compared with coronary computed tomography angiography, was independently associated with recurrent ASCVD events, while cIMT was not. Although several investigators have examined the predictive value of cIMT and other metrics obtained from carotid ultrasonography, few studies have specifically compared the prognostic value of cIMT and cPS. In the present study, we demonstrated that compared with cIMT, cPS may provide superior risk stratification in patients of a secondary 
prevention setting. It is not surprising that the calculation of cPS, which is a reflection of the cumulative atherosclerotic burden in the carotid artery, is superior in predicting future cardiovascular events compared with the calculation of IMT in the common carotid artery. Additionally, cIMT shows normal distribution, and even extremely low-risk individuals do not exhibit score of "zero". Moreover, the absolute difference between high-risk and low-risk individuals is small. On the other hand, cPS reflects the sum of the "plaque" whose intima-media thickening $\geq 1.1 \mathrm{~mm}$, leading to reflect higher risk situation compared to cIMT. This factor could contribute to enhance the power of cPS especially among the patients with secondary prevention setting. Additionally, cPS could also reflect lower risk situation because of the powerful negative predictive value ${ }^{9)}$. Those factors appear to collectively contribute to better risk discriminatory power of cPS, compared with cIMT. Moreover, cPS has been shown as an useful marker for good predictor of cardiovascular mortality even in the very old population ${ }^{20)}$. Accordingly, we suggest to assess cPS, rather than cIMT for the risk stratification and as a surrogate marker for the progression of atherosclerosis in patients of a secondary prevention settings, as suggested in the patients with $\mathrm{FH}$.

Based on regression lines from age and cPS among $\mathrm{FH}$ patients, we previously reported that carotid atherosclerosis may start to develop at 17 and 26 years of age in male and female $\mathrm{FH}$ patients, respectively ${ }^{8)}$. In this study, we performed the same analyses, and found that carotid atherosclerosis starts to develop at 27 and 32 years of age in male and female patients of a secondary prevention settings, respectively. These results suggest that carotid atherosclerosis might have already started to develop, at those early ages in particular high-risk population. Therefore, initiating lipid-lowering therapy should be considered before such ages in this high-risk population.

The present study had several limitations. First, this was a retrospective study with a small sample size. In addition, we could not include all patients who underwent carotid ultrasonography in the study due to the lack of data. Second, we did not account for medication information, which could affect cIMT and cPS. Third, our assumption of the development of carotid atherosclerosis in such patients is based on a linear model, which may not be applicable to those individuals. Further prospective studies accounting for those limitations are needed to validate our results.

\section{Acknowledgements and Notice of Grant Support}

We express special thanks to Ms. Kazuko Honda, Ms. Takako Terakami, Mr. Yoshiyasu Miyajima, and Mr. Sachio Yamamoto (staff of Kanazawa University) for their technical assistance in this study.

\section{Conflicts of Interest}

None.

\section{References}

1) Levine GN, Bates ER, Blankenship JC, Bailey SR, Bittl JA, Cercek B, Chambers CE, Ellis SG, Guyton RA, Hollenberg SM, Khot UN, Lange RA, Mauri L, Mehran R, Moussa ID, Mukherjee D, Ting HH, O'Gara PT, Kushner FG, Ascheim DD, Brindis RG, Casey DE Jr, Chung MK, de Lemos JA, Diercks DB, Fang JC, Franklin BA, Granger CB, Krumholz HM, Linderbaum JA, Morrow DA, Newby LK, Ornato JP, Ou N, Radford MJ, TamisHolland JE, Tommaso CL, Tracy CM, Woo YJ, Zhao DX. 2015 ACC/AHA/SCAI Focused Update on Primary Percutaneous Coronary Intervention for Patients With ST-Elevation Myocardial Infarction: An Update of the 2011 ACCF/AHA/SCAI Guideline for Percutaneous Coronary Intervention and the 2013 ACCF/AHA Guideline for the Management of ST-Elevation Myocardial Infarction: A Report of the American College of Cardiology/American Heart Association Task Force on Clinical Practice Guidelines and the Society for Cardiovascular Angiography and Interventions. Circulation, 2016; 133: 1135-1147

2) Grundy SM, Stone NJ, Bailey AL, Beam C, Birtcher KK, Blumenthal RS, Braun LT, Braun LT, de Ferranti S, Faiella-Tommasino J, Forman DE, Goldberg R, Heidenreich PA, Hlatky MA, Jones DW, Lloyd-Jones D, Lopez-Pajares N, Ndumele CE, Orringer CE, Peralta CA, Saseen JJ, Smith SC Jr, Sperling L, Virani SS, Yeboah J. 2018 AHA/ ACC/AACVPR/AAPA/ABC/ACPM/ADA/AGS/APhA/ ASPC/NLA/PCNA Guideline on the Management of Blood Cholesterol: Executive Summary: A Report of the American College of Cardiology/American Heart Association Task Force on Clinical Practice Guidelines. J Am Coll Cardiol, 2019; 73: 3168-3209

3) Levine GN, Bates ER, Bittl JA, Brindis RG, Fihn SD, Fleisher LA, Granger CB, Lange RA, Mack MJ, Mauri L, Mehran R, Mukherjee D, Newby LK, O'Gara PT, Sabatine MS, Smith PK, Smith SC Jr. 2016 ACC/AHA Guideline Focused Update on Duration of Dual Antiplatelet Therapy in Patients With Coronary Artery Disease: A Report of the American College of Cardiology/ American Heart Association Task Force on Clinical Practice Guidelines: An Update of the 2011 ACCF/AHA/ SCAI Guideline for Percutaneous Coronary Intervention, 2011 ACCF/AHA Guideline for Coronary Artery Bypass Graft Surgery, 2012 ACC/AHA/ACP/AATS/PCNA/ SCAI/STS Guideline for the Diagnosis and Management 
of Patients With Stable Ischemic Heart Disease, 2013 ACCF/AHA Guideline for the Management of ST-Elevation Myocardial Infarction, 2014 AHA/ACC Guideline for the Management of Patients With Non-ST-Elevation Acute Coronary Syndromes, and 2014 ACC/AHA Guideline on Perioperative Cardiovascular Evaluation and Management of Patients Undergoing Noncardiac Surgery. Circulation, 2016; 134: e123-155

4) Hodis HN, Mack WJ, Barth J. Carotid intima-media thickness as a surrogate end point for coronary artery disease. Circulation, 1996; 94: 2311-2312

5) Osawa K, Trejo MEP, Nakanishi R, McClelland RL, Blaha MJ, Blankstein R, McEvoy JW, Ceponiene I, Stein JH, Sacco RL, Polak JF, Budoff MJ. Coronary artery calcium and carotid artery intima-media thickness for the prediction of stroke and benefit from statins. Eur J Prev Cardiol, 2018; 25: 1980-1987

6) Kokubo Y, Watanabe M, Higashiyama A, Nakao YM, Nakamura F, Miyamoto Y. Impact of Intima-Media Thickness Progression in the Common Carotid Arteries on the Risk of Incident Cardiovascular Disease in the Suita Study. J Am Heart Assoc, 2018; 7. pii: e007720

7) Jeevarethinam A, Venuraju S, Dumo A, Ruano S, Rosenthal M, Nair D, Cohen M, Darko D, Lahiri A, Rakhit R. Usefulness of Carotid Plaques as Predictors of Obstructive Coronary Artery Disease and Cardiovascular Events in Asymptomatic Individuals With Diabetes Mellitus. Am J Cardiol, 2018; 121: 910-916

8) Tada H, Kawashiri MA, Okada H, Nakahashi T, Sakata K, Nohara A, Inazu A, Mabuchi H, Yamagishi M, Hayashi K. Assessments of Carotid Artery Plaque Burden in Patients With Familial Hypercholesterolemia. Am J Cardiol, 2017; 120: 1955-1960

9) Ikeda N, Kogame N, Iijima R, Nakamura M, Sugi K. Carotid artery intima-media thickness and plaque score can predict the SYNTAX score. Eur Heart J, 2012; 33: 113-119

10) Sillesen H, Sartori S, Sandholt B, Baber U, Mehran R, Fuster V. Carotid plaque thickness and carotid plaque burden predict future cardiovascular events in asymptomatic adult Americans. Eur Heart J Cardiovasc Imaging, 2018; 19: 1042-1050

11) Tada H, Kawashiri MA, Okada H, Teramoto R, Konno T, Yoshimuta T, Sakata K, Nohara A, Inazu A, Kobayashi J, Mabuchi H, Yamagishi M, Hayashi K. Assessment of coronary atherosclerosis in patients with familial hypercholesterolemia by coronary computed tomography angiography. Am J Cardiol, 2015; 115: 724-729

12) Tada H, Kawashiri MA, Yoshida T, Teramoto R, Nohara A, Konno T, Inazu A, Mabuchi H, Yamagishi M, Hayashi K. Lipoprotein(a) in familial hypercholesterolemia with proprotein convertase subtilisin/kexin type 9 (PCSK9) gain-of-function mutations. Circ J, 2016; 80: 512-518

13) Committee of the Japan Diabetes Society on the Diagnostic Criteria of Diabetes Mellitus, Seino Y, Nanjo K, Tajima N, Kadowaki T, Kashiwagi A, Araki E, Ito C, Inagaki N, Iwamoto Y, Kasuga M, Hanafusa T, Haneda M, Ueki K. Report of the committee on the classification and diagnostic criteria of diabetes mellitus. J Diabetes Investig, 2010; 1: 212-228

14) Nakade Y, Toyama T, Furuichi K, Kitajima S, Miyajima Y, Fukamachi M, Sagara A, Shinozaki Y, Hara A, Shimizu M, Iwata Y, Oe H, Nagahara M, Horita H, Sakai Y, Kaneko S, Wada T. Impact of kidney function and urinary protein excretion on intima-media thickness in Japanese patients with type 2 diabetes. Clin Exp Nephrol, 2015; 19: 909-917

15) Nakahashi T, Tada H, Sakata K, Nomura A, Ohira M, Mori M, Takamura M, Hayashi K, Yamagishi M, Kawashiri MA. Additive Prognostic Value of Carotid Plaque Score to Enhance the Age, Creatinine, and Ejection Fraction Score in Patients with Acute Coronary Syndrome. J Atheroscler Thromb, 2018; 25: 709-719

16) Morrow DA. Cardiovascular risk prediction in patients with stable and unstable coronary heart disease. Circulation, 2010; 121: 2681-2691

17) Antoni ML, Hoogslag GE, Boden H, Liem SS, Boersma E, Fox K, Schalij MJ, Bax JJ, Delgado V. Cardiovascular mortality and heart failure risk score for patients after STsegment elevation acute myocardial infarction treated with primary percutaneous coronary intervention (Data from the Leiden MISSION! Infarct Registry). Am J Cardiol, 2012; 109: 187-194

18) Battes L, Barendse R, Steyerberg EW, Simoons ML, Deckers JW, Nieboer D, Bertrand M, Ferrari R, Remme WJ, Fox K, Takkenberg JJ, Boersma E, Kardys I. Development and validation of a cardiovascular risk assessment model in patients with established coronary artery disease. Am J Cardiol, 2013; 112: 27-33

19) Goliasch G, Kleber ME, Richter B, Plischke M, Hoke M, Haschemi A, Marculescu R, Endler G, Grammer TB, Pilz S, Tomaschitz A, Silbernagel G, Maurer G, Wagner O, Huber K, März W, Mannhalter C, Niessner A. Routinely available biomarkers improve prediction of long-term mortality in stable coronary artery disease: the Vienna and Ludwigshafen Coronary Artery Disease (VILCAD) risk score. Eur Heart J, 2012; 33: 2282-2289

20) Hirata T, Arai Y, Takayama M, Abe Y, Ohkuma K, Takebayashi T. Carotid Plaque Score and Risk of Cardiovascular Mortality in the Oldest Old: Results from the TOOTH Study. J Atheroscler Thromb, 2018; 25: 55-64 


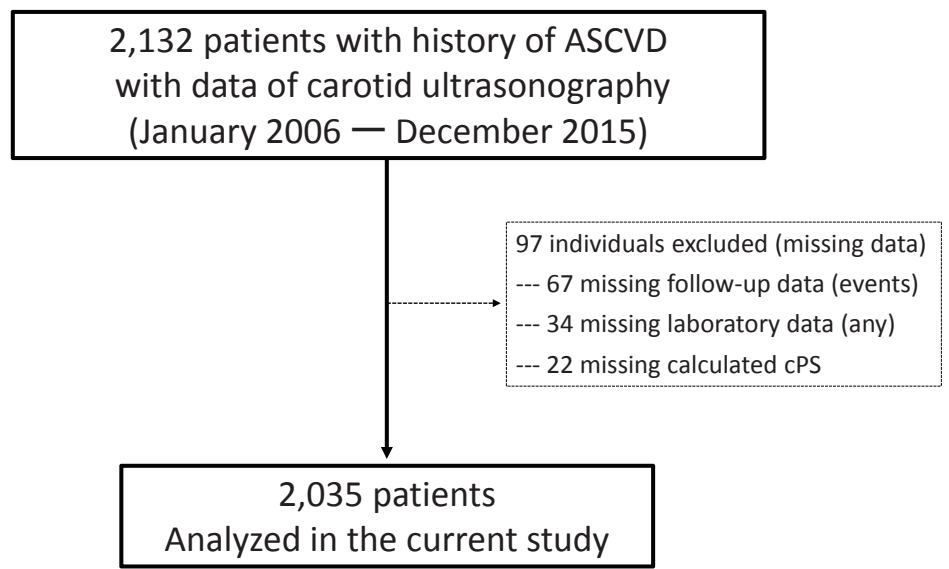

Supplemental Fig. 1. Flow chart of this study

In total, 2,132 consecutive patients who had any histories of ASCVD events and underwent carotid ultrasonography between January 2006 and December 2015 were retrospectively analyzed. Ninety seven patients lacking data (5\%) were excluded.
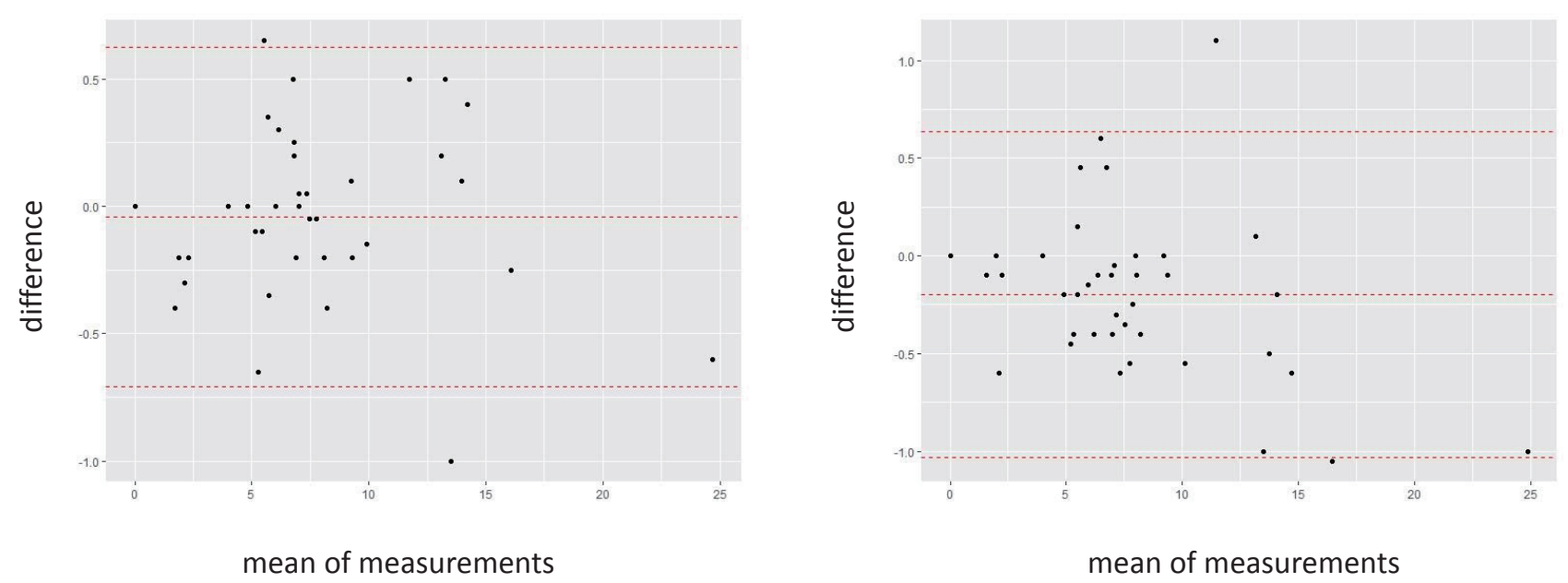

Supplemental Fig. 2. Bland-Altmann plot of cIMT

$\mathrm{X}$-axis represents mean of the difference. Y-axis represents difference. (A) Intraobserver. (B) Interobserver.
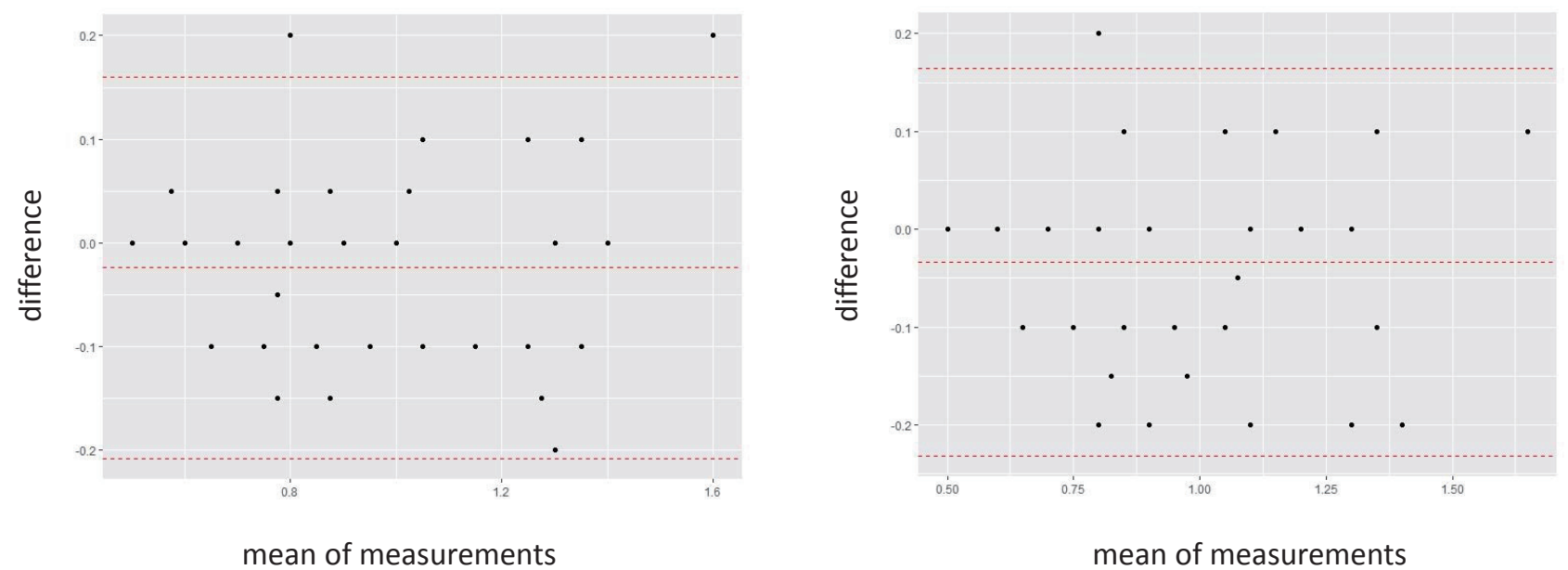

Supplemental Fig.3. Bland-Altmann plot of cPS

$\mathrm{X}$-axis represents mean of the difference. Y-axis represents difference. (A) Intraobserver. (B) Interobserver. 